\title{
SPINK1 is a prognosis predicting factor of non-small cell lung cancer and regulates redox homeostasis
}

\author{
MAOQING GUO ${ }^{1 *}$, XUAN ZHOU ${ }^{2 *}$, XIAO HAN ${ }^{1}$, YOUWEN ZHANG ${ }^{1}$ and LUNING JIANG ${ }^{1}$ \\ Departments of ${ }^{1}$ Respiratory Medicine and ${ }^{2}$ Critical Care Medicine, \\ Affiliated Hospital of Jining Medical University, Jining, Shandong 272029, P.R. China
}

Received January 26, 2019; Accepted September 11, 2019

DOI: $10.3892 / \mathrm{ol} .2019 .11005$

\begin{abstract}
Serine protease inhibitor Kazal-type 1 (SPINK1) is a trypsin kinase inhibitor, which is involved in the development of inflammation, cell proliferation and cancer development and progression. However, the prognostic value of SPINK1 in non-small cell lung cancer (NSCLC) and its ability to regulate intrinsic redox homeostasis have, to the best of our knowledge, not been previously investigated. In the present study, it was revealed that SPINK1 is highly expressed in NSCLC tissue samples compared with normal tissue samples, and may be a potential prognostic marker of NSCLC. Functional analyses demonstrated that SPINK1 promoted tumor cell growth and inhibited apoptosis through maintaining redox homeostasis by regulating the nuclear factor erythroid 2-related factor 2 pathways. It has been proposed that SPINK1 could be a prognostic marker of NSCLC and a novel antioxidant promoter under oxidative stress conditions in NSCLC.
\end{abstract}

\section{Introduction}

Lung cancer is the most commonly diagnosed malignancy and the main cause of cancer mortality worldwide, accounting for 1.6 million deaths per year (1). In particular, non-small cell lung cancer (NSCLC) accounts for $\sim 80 \%$ of lung cancer cases (2). Smoking and exposure to pollutants are key factors in NSCLC tumorigenesis (3). It has been suggested that cigarette and air pollutants promote the progression of cancer through oxidative stress in cells and tissues (4). Similarly, endogenous oxidants are formed

Correspondence to: Dr Xiao Han, Department of Respiratory Medicine, Affiliated Hospital of Jining Medical University, 89 Guhuai Road, Jining, Shandong 272029, P.R. China

E-mail: 383396405@qq.com

"Contributed equally

Key words: serine protease inhibitor Kazal-type 1, non-small cell lung cancer, redox homeostasis, nuclear factor erythroid 2-related factor 2 continuously (3). However, in the case of excess oxidants, reactive oxidative species (ROS) are generated and induce a redox imbalance, modifying numerous biomolecular markers $(5,6)$. Following this imbalance, ROS readily react with proteins, lipids and DNA resulting in a number of pathological consequences, including induced cell death, apoptosis and senescence, and an increased risk for developing cancer (7).

ROS have been reported to be involved in the pathogenesis of cancer $(8,9)$; the mechanism by which this occurs has been extensively investigated (10). Smoking and pollutants, as well as internal metabolism, may induce ROS production, while hypoxia may also induce ROS to promote tumor growth (11). Furthermore, alterations in the signaling pathways in cancer, such as integrin activation, are also caused by an increase in ROS production (12). A previous study reported that ROS prevent cancer progression by regulating cancer cell death (13). However, the molecular mechanism underlying oxidants in NSCLC remain unknown.

Serine protease inhibitor Kazal-type 1 (SPINK1), also known as pancreatic secretory trypsin inhibitor or pancreatic secretory trypsin inhibitor, is a trypsin kinase inhibitor that encodes a 56 amino acid secreted peptide (14). The normal function of SPINK1 is thought to be the inhibition of serine proteases, such as trypsin, involved in inflammation and cell proliferation (15). The altered expression of SPINK1 has been demonstrated to be associated with disease prognosis and has also been identified in different types of cancer, including lung, bladder, kidney, prostate, testis, ovary, cervix and breast cancers, and has been used in targeted therapy $(16,17)$. In the present study, it was revealed that SPINK1 may be a prognostic factor for NSCLC and that SPINK1 regulates redox homeostasis through the nuclear factor erythroid 2-related factor 2 (NRF2) pathways.

\section{Materials and methods}

Human tissue collection. Paraffin-embedded tissue samples, including tumor and adjacent tissues, were obtained from January 2010 to December 2018 at The Department of Pathology, The Affiliated Hospital of Jining Medical University (Jining, China). Written informed consent was obtained from the patients and the study was approved by The Affiliated Hospital of Jining Medical University. All 
patients were diagnosed and confirmed to have NSCLC by histology. Patients who had received radiotherapy or chemotherapy were excluded. In total, 100 patients were included, 49 male and 51 female, with a median age of 61 years (range, 35-81 years). Staging was based on the pathological findings according to the 7th edition of The American Joint Committee on Cancer guidelines (18). Overall survival time ranged from 6 to 98 months, with a median of 28 months. All patients were followed up by telephone. The follow-up period ceased in December 2018. In addition, liquid nitrogen frozen samples of NSCLC tissue samples and adjacent normal tissues ( $2 \mathrm{~cm}$ from the lesion) were obtained by surgical resection from 20 patients. These 20 patients were in addition to the 100 patients aforementioned, and included 15 males and 5 females, with a median age of 60 years (range, 37-74 years).

Immunohistochemical staining. Briefly, tissue section $(4 \mu \mathrm{m})$ were deparaffinized in xylene and rehydrated in a graded alcohol series (100, 95, 85 and $75 \%$ ethanol) and blocked with $3 \% \mathrm{H}_{2} \mathrm{O}_{2}$ for $15 \mathrm{~min}$ at room temperature. Antigen retrieval was performed after heating in citrate buffer at $98^{\circ} \mathrm{C}$ for $10 \mathrm{~min}$. The sections were incubated with an antibody against SPINK1 (1:100; catalog no. ab183034; Abcam) at $4^{\circ} \mathrm{C}$ overnight and with horseradish peroxidase universal immunoglobulin $\mathrm{G}$ secondary antibody (cat. no. sc69786; 1:1,000; Santa Cruz Biotechnology, Inc.) for $30 \mathrm{~min}$ at $37^{\circ} \mathrm{C}$. The signal was detected with $3,3^{\prime}$-diaminobenzidine solution using a light microscope (magnification, x200; IX71; Olympus Corporation). For SPINK1 protein, all visual fields were analyzed; the entire tissue specimen irrespective of its size was assessed, taking into account the percentage of positively stained cells and the intensity of staining.

Scoring of immunohistochemistry. Double-blind scoring was performed independently by two pathologists from the Department of Pathology, Affiliated Hospital of Jining Medical University. If different scores were assigned for the same sample, the sample was revaluated and, if needed, further discussed to determine a final score. SPINK1 expression was scored according to a previously described staining intensity and percentage of positive cells method (19). The percentage of positive cells was scored as follows: 0 , no positive cells; $1,1-10 \%$ positive cells; $2,11-50 \%$ positive cells; $3, \geq 51 \%$ positive cells. Staining intensity was scored as follows: 0 , no staining; 1 , weak staining; 2 , moderate staining; 3 , dark staining. Comprehensive score $=$ staining percentage $\mathrm{x}$ intensity. SPINK1 expression: $<2$ low expression, $\geq 2$ high expression.

Cells and RNA interference (RNAi). The HBE, H460, H1299 and A549 cell lines were obtained from The American Type Culture Collection. A549 cells were cultured in F-12K medium with $10 \%$ fetal bovine serum (FBS; Gibco; Thermo Fisher Scientific, Inc.) at $37^{\circ} \mathrm{C}$ in a humidified atmosphere with $5 \%$ $\mathrm{CO}_{2}$. The other cell lines were cultured in RPMI-1640 medium with $10 \% \mathrm{FBS}$ in a humidified atmosphere with $5 \% \mathrm{CO}_{2}$ at $37^{\circ} \mathrm{C}$, until they reached $100 \%$ confluence. Small interfering (si)RNA targeting SPINK1 (SPINK1-si1, sense 5'-AAGAAA
GAUGCCUGUUACCUU-3', antisense 5'-GGUAACAGG CAUCUUUCUUCU-3' and SPINK1-si2, sense 5'-AGUGUU ACCAGAUAGACUCAA-3', antisense 5'-GAGUCUAUCUGG UAACACUGG-3'; Shanghai GenePharma Co, Ltd.) was used to knockdown the expression of SPINK1. Negative control siRNA ( $4 \mu \mathrm{g}$; non-targeting) or SPINK1 siRNA (Shanghai GenePharma Co, Ltd.) were transiently transfected using $8 \mu 1$ Lipofectamine ${ }^{\circledR}$ RNAiMAX (Invitrogen; Thermo Fisher Scientific, Inc.). The transfection efficiency was determined by western blotting $24 \mathrm{~h}$ following transfection.

Cell proliferation and apoptosis. Proliferation assays were performed by seeding 4,500 cells into 96 -well plates and using the Cell Counting Kit-8 (CCK-8; Dojindo Laboratories), according to the manufacturer's protocol. The Annexin V-FITC/propidium iodide (PI) apoptosis detection kit I (BD Biosciences) was used to determine the level of apoptosis. Briefly, the collected cells were centrifuged at $\mathrm{x} 500 \mathrm{~g}$ for $5 \mathrm{~min}$ at room temperature and washed three times with PBS, and resuspended with $100 \mu 1$ binding buffer with $5 \mu \mathrm{l}$ Annexin V-FITC and $5 \mu \mathrm{l}$ PI solution. Cells were incubated in the dark for $15 \mathrm{~min}$ at $4^{\circ} \mathrm{C}$. The cells were added to $400 \mu \mathrm{l}$ binding buffer and analyzed using a FACS LSR II (BD Biosciences) flow cytometer, and analyzed using CellQuest software (version 5.1; BD Biosciences).

Western blot analysis. The protein levels of SPINK1 from cell lines were determined using western blot analysis. Briefly, proteins were lysed in RIPA (Beyotime Institute of Biotechnology). Protein concentration was determined using a bicinchoninic acid assay kit (Beyotime institute of Biotechnology). Protein $(20 \mu \mathrm{g})$ were separated by $10 \%$ SDS-PAGE and transferred onto a nitrocellulose membrane. After the transfer, 5\% bovine serum albumin (Thermo Fisher Scientific, Inc.) was used to block the membranes for $1 \mathrm{~h}$ at room temperature. The following primary antibodies were incubated overnight at $4^{\circ} \mathrm{C}$ : SPINK1 $(1: 1,000$; catalog no. ab183034; Abcam), $\beta$-actin (1:5,000; cat. no. ab8226; Abcam). Membranes were washed with TBST three times. The secondary antibody anti-mouse (1:1,000; cat. no. 7076; Cell Signaling Technology, Inc.) and anti-rabbit (1:1,000; cat. no. 7074; Cell Signaling Technology, Inc.) was incubated for $1 \mathrm{~h}$ at room temperature. Protein bands were visualized using ECL reagent (Thermo Fisher Scientific, Inc.), images were captured using a Tanon detection system, and densitometry analysis was performed using ImageQuant TL software version 1.1 (GE Healthcare Life Sciences).

Reverse transcription-quantitative ( $R T-q P C R$ ). Total RNA from cells or patients' tissues was extracted using TRIzol ${ }^{\mathbb{}}$ reagent (Invitrogen; Thermo Fisher Scientific, Inc.). The PrimeScript RT reagent kit (Takara Bio, Inc.) was used to synthesize complementary DNA, which was used in the qPCR (SYBR Green PCR kit; Takara Bio, Inc.) with a 7500 Fast Real-time PCR system (Applied Biosystems; Thermo Fisher Scientific, Inc.). The RT reactions were performed as follows: $37^{\circ} \mathrm{C}$ for $15 \mathrm{~min}$ and $85^{\circ} \mathrm{C}$ for $5 \mathrm{sec}$. PCR amplification was performed in a thermal cycler for 40 cycles at the following cycle conditions: $95^{\circ} \mathrm{C}$ for $5 \mathrm{sec}, 60^{\circ} \mathrm{C}$ for $30 \mathrm{sec}$, and $72^{\circ} \mathrm{C}$ for $30 \mathrm{sec}$. The following primers were used: SPINK1, forward 
5'-TGTCTGTGGGACTGATGGAA-3', reverse 5'-AGGCCC AGATTTTTGAATGA-3'. NRF2, forward 5'-AGGTTGCCC ACATTCCCAAA-3', reverse 5'-AGTGACTGAAACGTA GCCGA-3'; ME1, forward 5'-GAACCCTCACCTCAACAA GGTA-3', reverse 5'-AGCCAAGAATACGCTCTCCA-3'; TXNRD1, forward 5'-GAAAGGCAAGAACGGCGATG-3', reverse 5'-CGTGTGCATGTGGACCTACT-3'; GCLC, forward 5'-ACTTCATTTCCCAGTACCTTAACA-3', reverse 5'-CCG GCTTAGAAGCCCTTGAA-3'; GCLM, forward 5'-AAGCAC TTTCTCGGCTACGA-3', reverse 5'-GCGGGAGAGCTG ATTCCAAA-3'; and GAPDH, forward 5'-AGAAGGCTG GGGCTCATTTG-3' and reverse 5'-AGGGGCCATCCACAG TCTTC-3'. GAPDH was used as the internal control. Each experiment was performed in triplicate. The relative mRNA expression of each gene was calculated with the comparative quantitation cycle method $2^{-\Delta \Delta c q}(20,21)$.

ROS evaluation. Dichlorofluorescin diacetate (DCFH-DA; Beyotime Institute of Biotechnology) staining was used to detect the cytoplasmic ROS level. Briefly, cells were washed twice with PBS and incubated with $10 \mu \mathrm{M}$ DCFH-DA at $37^{\circ} \mathrm{C}$ for $30 \mathrm{~min}$. The cells were resuspended in ice-cold PBS and the fluorescence intensity of DCF was determined at an excitation wavelength of $488 \mathrm{~nm}$ and an emission wavelength at $530 \mathrm{~nm}$.

The intracellular ATP level, NADPH/NADP ${ }^{+}$ratio and $\mathrm{NADH} / \mathrm{NAD}^{+}$ratio was evaluated using the ATP Assay kit (Beyotime Institute of Biotechnology), the NADP/NADPH Quantitation Colorimetric kit (BioVision) and the NAD/NADH Quantitation Colorimetric kit (BioVision), according to the manufacturers' protocols. The GSH and GSSG Assay kit was purchased from Beyotime Institute of Biotechnology and was performed according to the manufacturer's protocol.

Dual-luciferase reporter assay. In total, $2 \times 10^{4}$ cells were seeded into 96-well plates. The plasmids $(1 \mu \mathrm{g})$ REPO-ARE [a DNA sequence containing the GST A1 ARE (-833 to -533 from the start codon) inserted into a luciferase reporter vector as described previously (22)] and $0.5 \mu \mathrm{g} / 1 \mu \mathrm{g}$ pCMV-SPINK1-flag (both from Shanghai GenePharma Co, Ltd.) were transfected into each well using Lipofectamine ${ }^{\mathrm{TM}}$ 2000 (Invitrogen; Thermo Fisher Scientific, Inc.). The Renilla luciferase plasmid (Promega Corporation) was transfected into each well to normalize transfection efficiency. Luciferase activity (Dual-Luciferase Assay System; Promega Corporation) was detected $48 \mathrm{~h}$ after transfection. Renilla luciferase gene was used as the internal reference to verify the transfection efficiency and calculate the relative luciferase activity as follows: Relative luciferase activity=firefly luciferase activity/Renilla luciferase activity. The experiments were performed twice.

Statistical analysis. The data are presented as the mean \pm standard deviation. For comparisons between two groups, two-tailed Student's t-tests were used. Multiple group comparisons were calculated using the one-way ANOVA analysis followed by the Dunnett's post hoc test. Associations between the expression of SPINK1 and clinical characteristics were analyzed using the $\chi^{2}$ test. The Kaplan-Meier method and log-rank test was used to estimate overall survival (OS).
Table I. Comparisons with SPINK1 expression between NSCLC and paired adjacent normal tissues.

\begin{tabular}{lcccc}
\hline & & \multicolumn{2}{c}{$\begin{array}{c}\text { SPINK1 } \\
\text { expression }\end{array}$} & \\
\cline { 3 - 4 } Tissues & Cases, $\mathrm{n}$ & Low & High & P-value \\
\hline NSCLC tissues & 100 & 49 & 51 & 0.006 \\
Adjacent tissue & 100 & 68 & 32 & \\
\hline
\end{tabular}

SPINK1, serine protease inhibitor Kazal-type 1; NSCLC, non-small cell lung cancer.

Univariate and multivariate COX regression analysis was performed to estimate the relative risk of death associated with SPINK1 expression and other prognostic variables for OS. Those parameters with $\mathrm{P}<0.05$ in the univariate analyses were included in a Cox multivariate proportional hazards regression model. $\mathrm{P}<0.05$ was considered to indicate a statistically significant difference.

\section{Results}

SPINK1 is a prognostic marker of NSCLC. In total, 100 paraffin-embedded tissue samples used for detecting protein expression by IHC were collected in order to investigate the expression and clinical features of SPINK1 in patients with NSCLC in the present study. SPINK1 was expressed at a higher level in tumor samples compared with adjacent normal tissue (Table I). No differences in SPINK1 expression were observed for age, sex, smoking habits, histological type or the Tumor (T) and Node (N) stage (Table II). However, SPINK1 expression was associated with Tumor-Node-Metastasis (TNM) stage $(\mathrm{P}<0.001$; Table II). The univariate and multivariate analysis indicated that, in addition to TNM stage, SPINK1 may be a prognostic factor for patients with NSCLC $(\mathrm{P}<0.001$; Table III).

SPINK1 is expressed at a higher level in NSCLC. SPINK1 expression was analyzed in clinical samples. The function of SPINK1 was also analyzed. SPINK1 expression was higher in NSCLC tissues compared with adjacent normal tissues $(\mathrm{P}<0.05$; Fig. 1A). The expression of SPINK1 was also assessed in cell lines. SPINK1 was upregulated in NSCLC cell lines compared with $\mathrm{HBE}$ at both the transcriptional $(\mathrm{P}<0.05$; Fig. $1 \mathrm{~B})$ and protein levels (Fig. 1C and D). Immunohistochemistry demonstrated a higher level of expression of SPINK1 in NSCLC tissue samples (Fig. 1E). Kaplan-Meier analysis indicated that higher expression of SPINK1 predicted a poorer prognosis for patients $(\mathrm{P}<0.001$; Fig. 1F).

SPINK1 regulates proliferation and apoptosis in NSCLC. In order to investigate the biological effects of SPINK1 in NSCLC cells, SPINK1 was depleted using RNAi. The knockdown efficiency was determined using RT-PCR and western blotting ( $\mathrm{P}<0.05$; Fig. $2 \mathrm{~A}$ and $\mathrm{B})$. The biological function of SPINK1 was examined in NSCLC cell lines. The viability 
Table II. Association between the clinicopathological characteristics and SPINK1 expression in non-small cell lung cancer.

SPINK1 expression

\begin{tabular}{|c|c|c|c|c|}
\hline Characteristics & $\mathrm{N}$ & Low $(n=49)$ & $\operatorname{High}(n=51)$ & P-value \\
\hline \multicolumn{5}{|l|}{ Age, years } \\
\hline$\leq 60$ & 50 & 22 & 28 & 0.317 \\
\hline$>60$ & 50 & 27 & 23 & \\
\hline \multicolumn{5}{|l|}{ Sex } \\
\hline Male & 55 & 26 & 29 & 0.702 \\
\hline Female & 45 & 23 & 22 & \\
\hline \multicolumn{5}{|l|}{ Smoking habit } \\
\hline Never & 64 & 31 & 33 & 0.881 \\
\hline Ever & 36 & 18 & 18 & \\
\hline \multicolumn{5}{|l|}{ Histological type } \\
\hline Adenocarcinoma & 46 & 25 & 21 & 0.319 \\
\hline Squamous cell carcinoma & 36 & 14 & 22 & \\
\hline Others & 18 & 10 & 8 & \\
\hline \multicolumn{5}{|l|}{ Grade } \\
\hline Well-differentiated & 70 & 36 & 34 & 0.458 \\
\hline Moderate-poorly differentiated & 30 & 13 & 17 & \\
\hline \multicolumn{5}{|l|}{ T stage } \\
\hline $\mathrm{T} 1+\mathrm{T} 2$ & 30 & 14 & 16 & 0.711 \\
\hline $\mathrm{T} 3$ & 69 & 35 & 34 & \\
\hline \multicolumn{5}{|l|}{ N stage } \\
\hline No & 39 & 22 & 17 & 0.236 \\
\hline $\mathrm{N} 1+\mathrm{N} 2$ & 61 & 27 & 34 & \\
\hline \multicolumn{5}{|l|}{ TNM stage } \\
\hline $\mathrm{I}+\mathrm{II}$ & 66 & 42 & 24 & $<0.001$ \\
\hline IIIA & 34 & 7 & 27 & \\
\hline
\end{tabular}

SPINK1, serine protease inhibitor Kazal-type1; T, tumor; N, node; TNM, Tumor-Node-Metastasis.

of SPINK1-siRNA treated cells was significantly decreased compared with the control group $(\mathrm{P}<0.05$; Fig. $2 \mathrm{C}$ and $\mathrm{D})$. In addition, the level of apoptosis was higher in SPINK1-siRNA treated cells $(\mathrm{P}<0.05$; Fig. $2 \mathrm{E}$ and $\mathrm{F})$. These results indicated a role for SPINK1 in promoting cell growth and inhibiting apoptosis.

SPINK1 regulates the redox state of NSCLC cells. The potential molecular mechanisms of SPINK1 in NSCLC were investigated in the present study. Redox homeostasis is an important factor in tumor progression $(23,24)$. Therefore, whether SPINK1 is as an oxidative factor involved in protecting basal mitochondrial respiration in NSCLC cells was investigated in the present study. Since A549 and H1299 have relatively higher endogenous SPINK1 expression, these cell lines were chosen for SPINK1 silencing experiments. The results revealed that the inhibition of SPINK1 significantly increased ROS production. Furthermore, the knockdown of SPINK1 increased the NADP/NADPH ratio, decreased the GSH/GSSG ratio and JC-1 aggregates compared with the control cells $(\mathrm{P}<0.05$; Fig. $3 \mathrm{~A}$ and $\mathrm{B})$. These results suggested that SPINK1 played an important role in the redox balance of NSCLC cells.

SPINK1 transcriptionally activates NRF2 expression in NSCLC. Next, the potential underlying molecular mechanism of SPINK1 regulating redox homeostasis was investigated. Antioxidant response elements (AREs) are in the regulatory regions of several genes that encode for enzymes that contribute to the regulation of antioxidants (21). The results of the present study revealed that ARE activity was increased in a dose-dependent manner after cloning the ARE sequences into a luciferase vector and transfecting cells with a SPINK1 expression vector, indicating that ARE-driven gene were significantly decreased in SPINK1 knockdown cells $(\mathrm{P}<0.05$; Fig. 4A and B). The knockdown of SPINK1 decreased NRF2 expression and further decreased the mRNA levels of ARE-driven genes, including NADP-dependent malic enzyme (ME1), thioredoxin reductase 1 (TXNRD), glutamate-cysteine ligase catalytic subunit (GCLC) and glutamate-cysteine ligase regulatory subunit (GCLM) ( $\mathrm{P}<0.05$; Fig. 4C and D). Furthermore, the expression levels of NRF2, ME1, TXNRD 1, 
Table III. Summary of univariate and multivariate Cox regression analysis of overall survival.

\begin{tabular}{|c|c|c|c|c|c|c|}
\hline \multirow[b]{2}{*}{ Characteristics } & \multicolumn{3}{|c|}{ Univariate analysis } & \multicolumn{3}{|c|}{ Multivariate analysis } \\
\hline & HR & $95 \% \mathrm{CI}$ & P-value & HR & $95 \% \mathrm{CI}$ & P-value \\
\hline \multicolumn{7}{|l|}{ SPINK1 expression } \\
\hline Low & 1 & & & 1 & & \\
\hline High & 4.003 & $2.450-6.541$ & $<0.001$ & 3.491 & $2.071-5.884$ & $<0.001$ \\
\hline \multicolumn{7}{|l|}{ Age, years } \\
\hline$\leq 60$ & 1 & & & & & \\
\hline$>60$ & 0.821 & $0.536-1.256$ & 0.362 & & & \\
\hline \multicolumn{7}{|l|}{ Sex } \\
\hline Male & 1 & & & & & \\
\hline Female & 0.868 & $0.566-1.331$ & 0.517 & & & \\
\hline \multicolumn{7}{|l|}{ Smoking habit } \\
\hline Never & 1 & & & & & \\
\hline Ever & 0.947 & $0.610-1.471$ & 0.947 & & & \\
\hline \multicolumn{7}{|l|}{ Histological type } \\
\hline Adenocarcinoma & 1 & & & & & \\
\hline Squamous cell carcinoma & 0.970 & $0.536-1.758$ & 0.921 & & & \\
\hline Other & 1.362 & $0.729-2.542$ & 0.332 & & & \\
\hline \multicolumn{7}{|l|}{ Grade } \\
\hline Well differentiated & 1 & & & & & \\
\hline Moderate-poorly differentiated & 1.500 & $0.951-2.368$ & 0.081 & & & \\
\hline \multicolumn{7}{|l|}{ T stage } \\
\hline $\mathrm{T} 1+\mathrm{T} 2$ & 1 & & & & & \\
\hline $\mathrm{T} 3$ & 0.731 & $0.416-1.284$ & 0.275 & & & \\
\hline \multicolumn{7}{|l|}{$\mathrm{N}$ stage } \\
\hline N0 & 1 & & & & & \\
\hline $\mathrm{N} 1+\mathrm{N} 2$ & 0.858 & $0.684-1.076$ & 0.186 & & & \\
\hline \multicolumn{7}{|l|}{ TNM stage } \\
\hline I+II & 1 & & & 1 & & \\
\hline IIIA & 19.263 & $8.932-41.542$ & $<0.001$ & 19.117 & $8.243-44.333$ & $<0.001$ \\
\hline
\end{tabular}

SPINK1, serine protease inhibitor Kazal-type1; T, tumor; N, node; TNM, Tumor-Node-Metastasis.

GCLC and GCLM were validated using 20 NSCLC specimens frozen in liquid nitrogen. The results revealed that the expression levels of NRF2, ME1, TXNRD 1, GCLC and GCLM were increased in NSCLC tissues (in 18 of the 20 cases; $\mathrm{P}<0.05$; Fig. 4E). SPINK1 maintained the redox balance by activating NRF2, upregulating antioxidant response element-driven gene expression, leading to increased cell proliferation and decreased cell apoptosis (Fig. 4F).

\section{Discussion}

The serine protease inhibitor SPINK1 is a trypsin inhibitor, which is known to play an important role in a number of physiological and pathological processes (25). SPINK1 has an established function in protecting the pancreas from premature activation of trypsinogen (26). SPINK1 functions as an acute phase reactant in severe inflammatory disease and tissue injury, contributing to normal tissue maintenance and repair (14). Furthermore, SPINK1 promotes the initiation and progression of cancer. Changes in SPINK1 expression levels are associated with the prognosis of a number of different types of cancer (17); the high expression of SPINK1 in liver tissue is associated with liver metastasis (27), and SPINK1 levels in the serum of patients with colorectal cancer are associated with OS (28).

In the present study, SPINK1 expression levels were determined in NSCLC. The expression of SPINK1 was higher in tumor samples and lower in the adjacent normal tissue. Clinicopathological analysis revealed that SPINK1 expression was associated with TNM stage. The univariate and multivariate analyses indicated that SPINK1 may be a prognostic factor in patients with NSCLC. The Kaplan-Meier analysis suggested that higher expression levels of SPINK1 predicted a poorer OS time. 


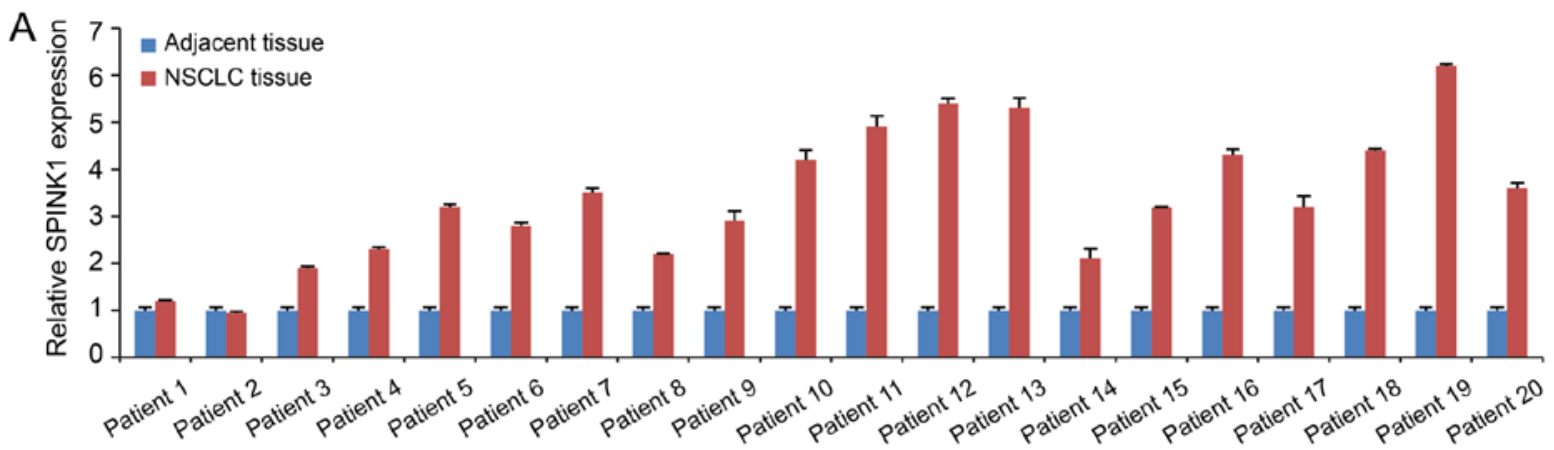

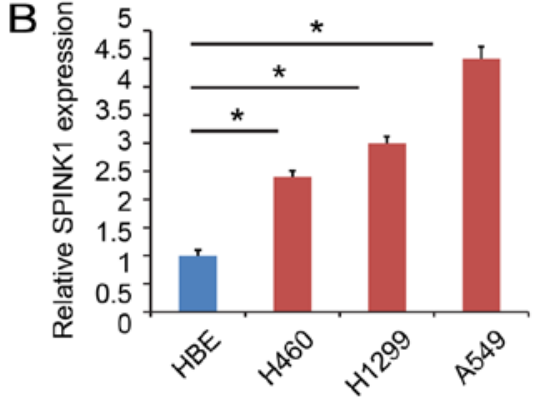

E

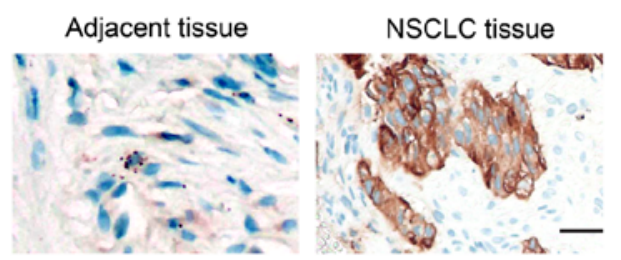

C

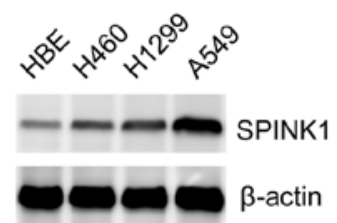

D

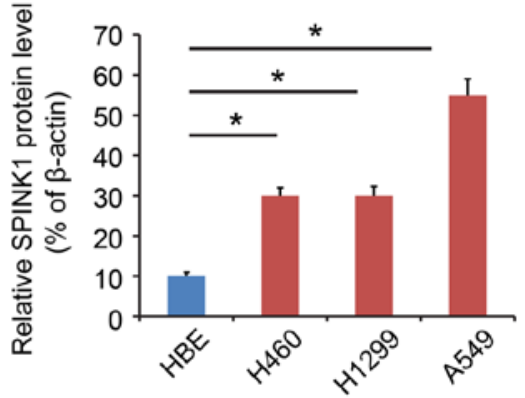

$\mathrm{F}$

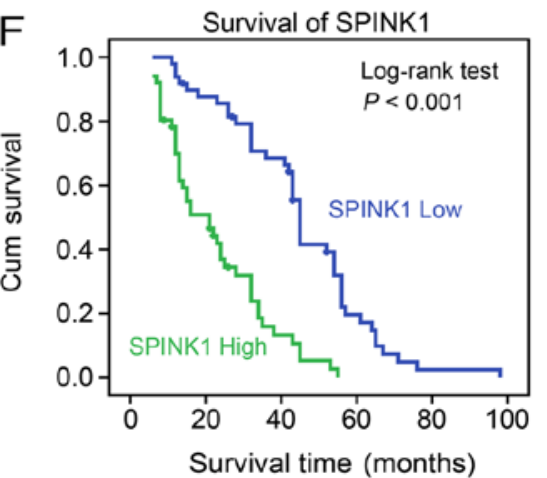

Figure 1. SPINK1 is expressed at a higher level in NSCLC. (A) SPINK1 expression is higher in tumor samples compared with adjacent normal tumor samples. (B) The mRNA expression of SPINK1 in cell lines. SPINK1 was upregulated in NSCLC cell lines compared with HBE. (C) The protein level of SPINK1 in NSCLC cell lines. SPINK1 was upregulated in NSCLC cell lines compared with HBE. (D) Quantification of SPINK1 expression. (E) Representative images of IHC staining of SPINK1 in NSCLC and adjacent tissues. IHC showed a higher level of SPINK1 expression in NSCLC tissue samples (brown color). (F) Kaplan-Meier analysis indicated a higher level of SINK1 expression predicted a poorer overall survival time. ${ }^{*} \mathrm{P}<0.05$ vs. control. IHC, immunohistochemistry; SPINK1, serine protease inhibitor Kazal-type 1; NSCLC, non-small cell lung cancer.

The biological functions of SPINK1 were also investigated in the present study. SPINK1 was highly expressed in NSCLC cells and tissue samples. The viability of SPINK1-siRNA treated cells was significantly attenuated compared with the control group. In addition, an increased level of apoptosis was observed in SPINK1-siRNA treated cells.

The molecular mechanism of SPINK1 in NSCLC was investigated in the present study. ROS have several roles in the development of tumors; the physiological effects of ROS vary with concentration, duration and cell changes (29). Low concentrations of ROS can accelerate cell division and proliferation, while moderate levels cause the arrest of cell growth and high levels induce apoptosis or necrosis (30). A large increase in ROS leads to the opening of the mitochondrial membrane permeability transport pores, activation of the caspase cascade, DNA breaks and tumor cell apoptosis mediated by Fas-FasL through activating the p38 mitogen-activated protein kinase (MAPK) signaling pathway $(31,32)$. ROS cause DNA damage (33), affecting mitochondrial DNA (mtDNA) and impeding oxidative phosphorylation (34). Previous studies have demonstrated that tumors, such as lung cancer, stomach cancer, breast cancer, colon cancer and lymphoma, have mtDNA mutations (35-38). In addition, low levels of ROS act as signaling molecules to regulate key proteins and promote cell proliferation. In proliferation, ROS leads to the decreased expression of p53 and p21, and increased activity of the cyclin-cyclin dependent kinase complex, leading to cell cycle progression $(39,40)$. In NSCLC, the inhibition of SPINK1 significantly increased ROS production, increased the NADPH/NADPH ratio and decreased the GSH/GSSG ratio, and decreased JC-1 aggregates. These results suggested that SPINK1 played an important role in the redox balance of NSCLC cells.

Previous studies have revealed that the NRF2 pathway is associated with ROS (41-44). AREs are found in the regulatory regions of antioxidant regulating enzymes (45). Following the transfection of the luciferase vector with ARE sequences and the SPINK1 vector in the present study, ARE activity increased in a dose-dependent manner. In addition, SPINK1 increased the expression of NRF2 and increased the mRNA levels of ARE-driven genes, including ME1, TXNRD, GCLC 

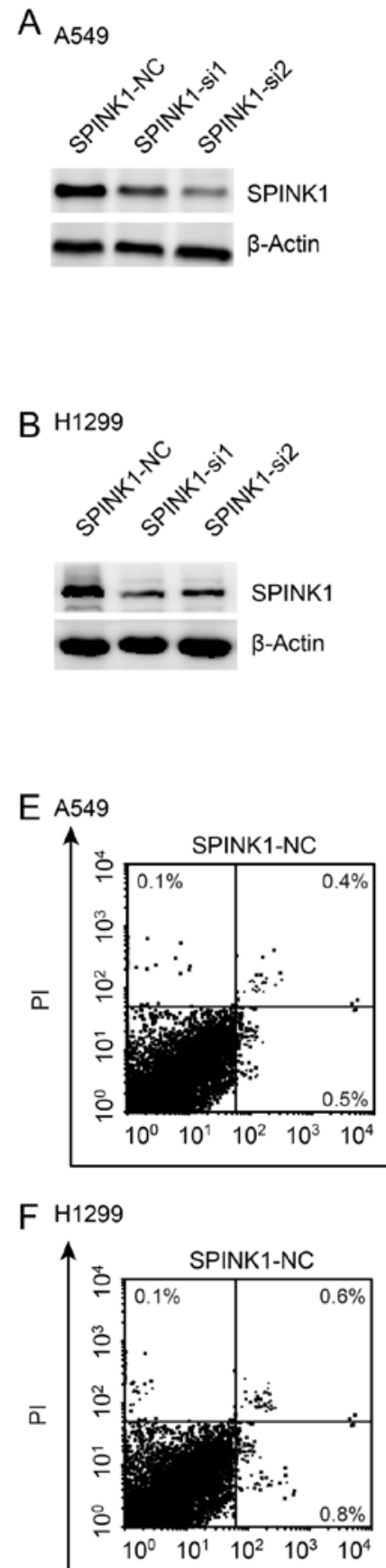
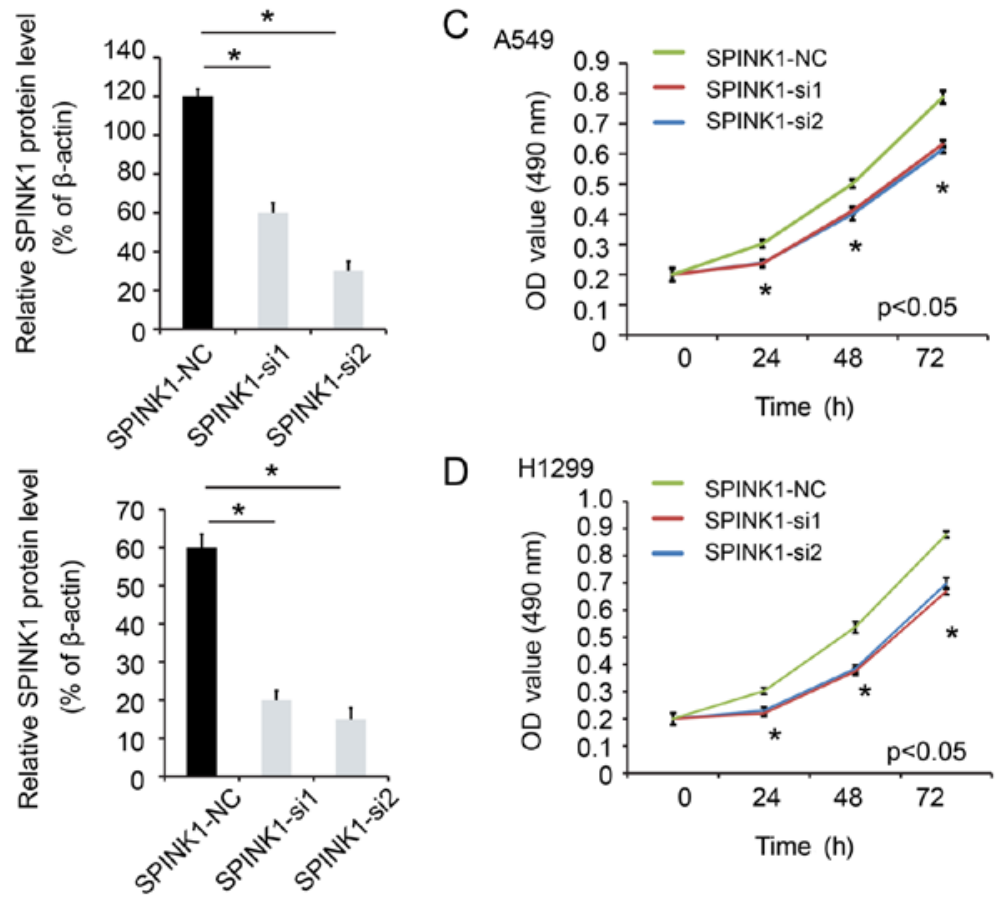

D $\mathrm{H} 1299$

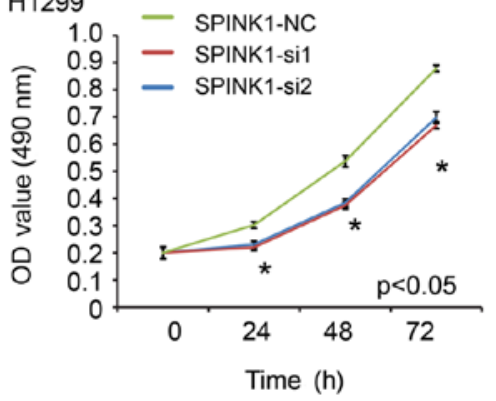

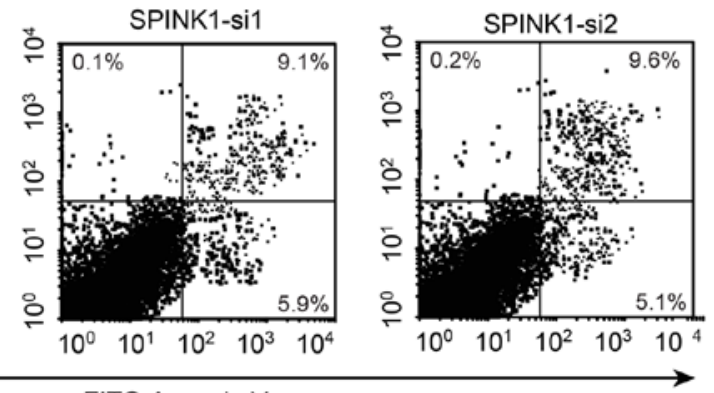

FITC-Annexin V

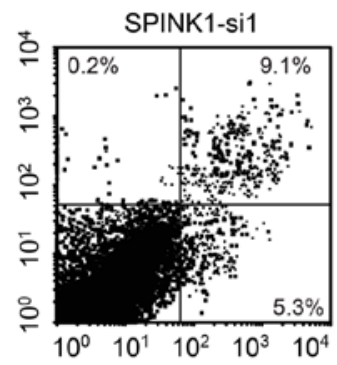

FITC-Annexin V
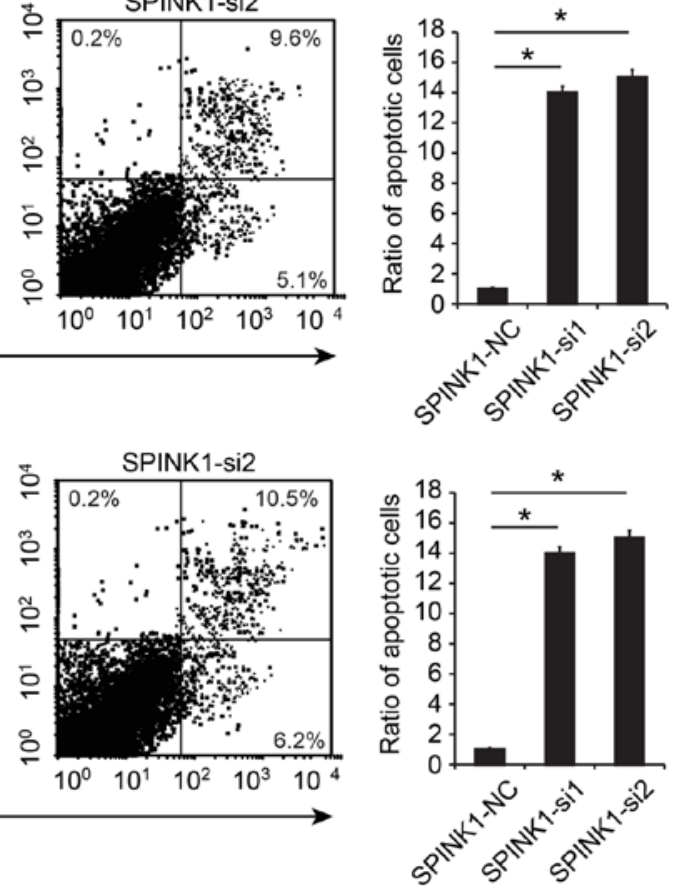

Figure 2. SPINK1 regulates the proliferation and apoptosis of NSCLC. (A and B) Protein expression of SPINK1 following siRNA transfection using western blotting. Quantification of SPINK1 expression. (C and D) The viability of SPINK1-siRNA transfected cells was significantly attenuated compared with the control group. (E and F) The level of apoptosis of SPINK1-siRNA treated cells and the control group. "P<0.05 vs. control. SPINK1, serine protease inhibitor Kazal-type 1; NSCLC, non-small cell lung cancer; siRNA, small interfering RNA; NC, negative control; OD, optical density.

and GCLM. Taken together, SPINK1 participated in the redox balance by activating NRF2 in NSCLC cells. However, how SPINK1 regulates NRF2 requires further investigation. Structurally, SPINK1 was identified as having a structure with $\sim 50 \%$ similarity to epidermal growth factor (EGF), which indicates the ability of SPINK1 to induce the dimerization and phosphorylation of the EGF receptor (EGFR), and promote signal transduction and cell proliferation through the MAPK pathway (46). Wang et al (47) reported that SPINK1 promotes the epithelial-to-mesenchymal transition through the EGFR signaling pathway in prostate cancer. The concomitant expression of SPINK1 at high levels and EGFR was also identified to be associated with poor prognosis in colorectal cancer (48). Ateeq et al (49) suggested that SPINK1 functions, at least in part, to stimulate EGFR signaling in an autocrine loop. A direct interaction between SPINK1 and EGFR was also identified in prostate cancer. Future research in the field should focus on this.

In conclusion, the present study revealed a novel regulatory function for SPINK1 through the activation of the NRF2-mediated antioxidant regulation. In addition, SPINK1 

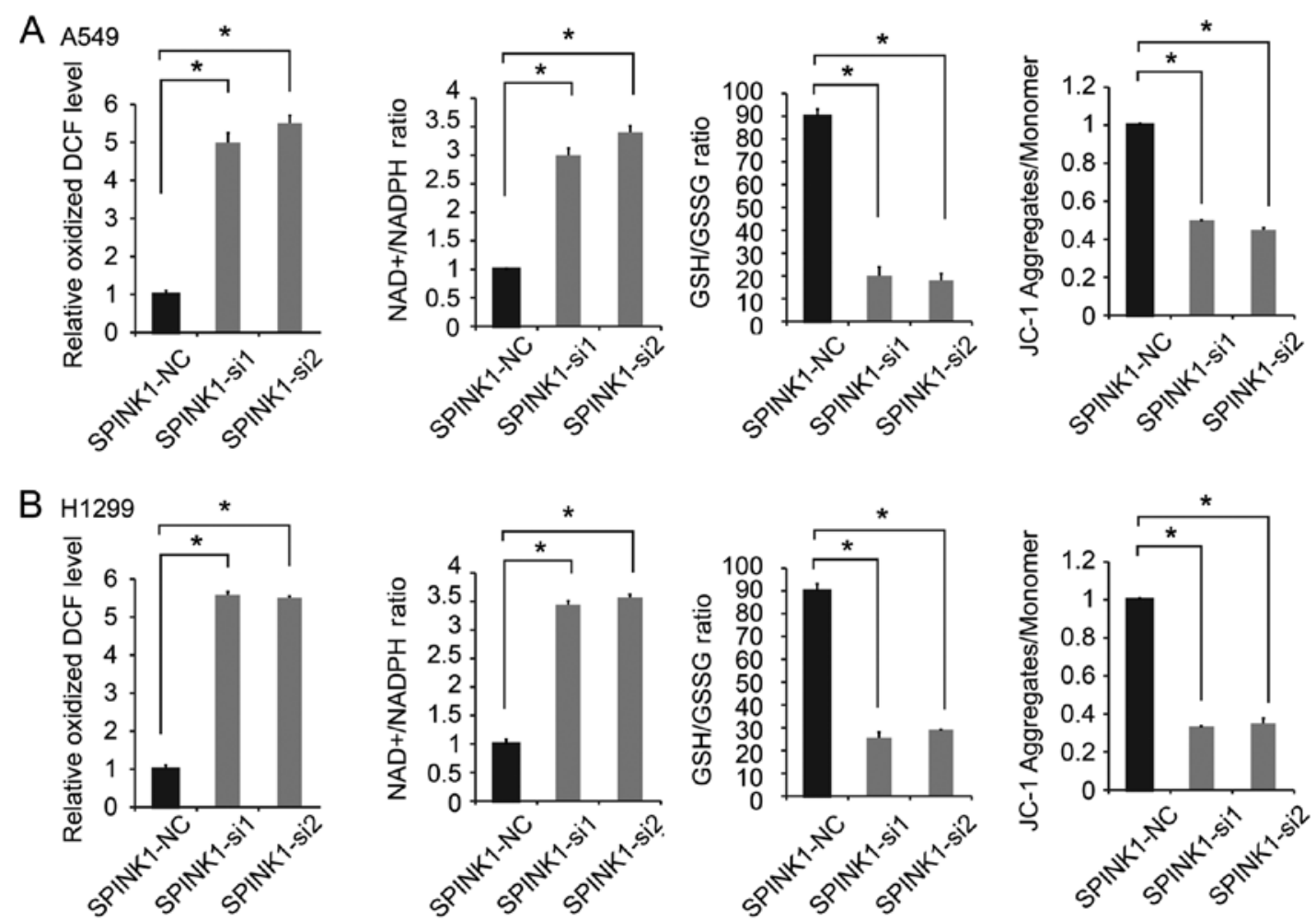

Figure 3. SPINK1 regulates the redox status of NSCLC cells. The inhibition of SPINK1 expression significantly increased oxidized DCF level (ROS production), increased the NADP/NADPH ratio, decreased GSH/GSSG ratio and decreased JC-1 aggregates in (A) A549 and (B) H1299 cells. "P<0.05 vs. control. SPINK1, serine protease inhibitor Kazal-type 1; NSCLC, non-small cell lung cancer; ROS, reactive oxygen species.
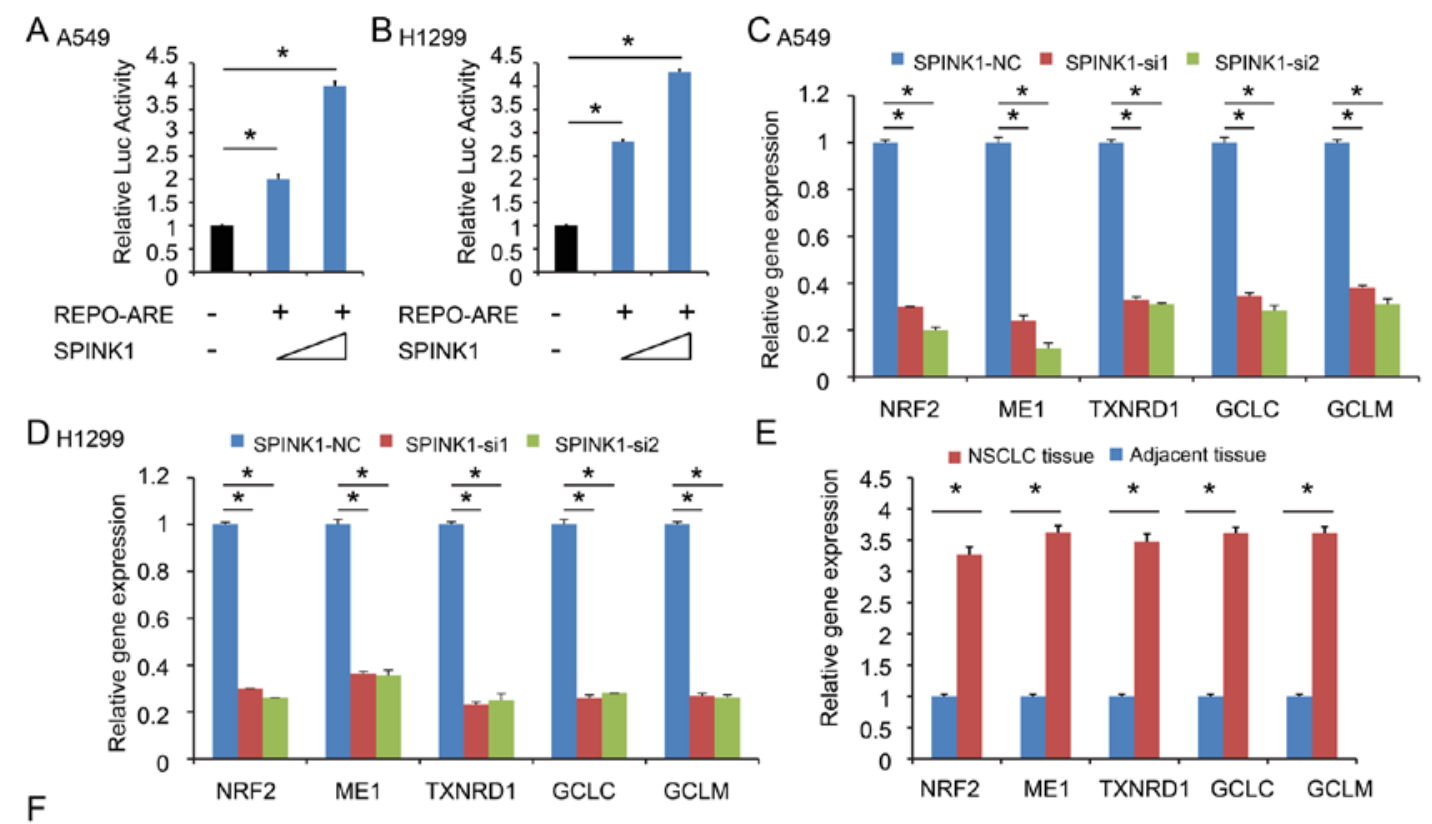

$\mathrm{F}$

$$
\left.\mathrm{SPINK1} \rightarrow \begin{array}{l}
\text { NRF2 } \\
\text { pathway }
\end{array} \rightarrow \begin{array}{l}
\text { ME1 } \\
\text { TXNRD1 } \\
\text { GCLC } \\
\text { GCLM }
\end{array}\right] \text { ROS production } \leadsto \begin{aligned}
& \text { Increased } \\
& \text { cell proliferation } \\
& \text { Decreased } \\
& \text { cell apoptosis }
\end{aligned}
$$

Figure 4. SPINK1 activates the transcription of NRF2 in NSCLC. (A and B) ARE activity was increased in a dose-dependent manner after cloning of the ARE sequences into a luciferase vector and co-transfection with a SPINK1 expression vector. (C and D) SPINK1 increased the expression of NRF2, ME1, TXNRD, GCLC and GCLM. (E) NRF2, ME1, TXNRD1, GCLC and GCLM were upregulated in NSCLC tissues. (F) The outline of the mechanism for SPINK1 mediated redox regulation in NSCLC. ${ }^{*} \mathrm{P}<0.05$ vs. control. ARE, antioxidant response element; SPINK1, serine protease inhibitor Kazal-type 1; NSCLC, non-small cell lung cancer; ARE, antioxidant response element; NRF2, nuclear factor erythroid 2-related factor; ME1, NADP-dependent malic enzyme; TXNRD, thioredoxin reductase 1; GCLC, glutamate-cysteine ligase catalytic subunit; GCLM, glutamate-cysteine ligase regulatory subunit. 
may be a prognostic factor for NSCLC. Thus, developing an inhibitor for SPINK1 is expected to provide therapeutic benefits for NSCLC.

\section{Acknowledgements}

The authors would like to thank Dr Ting Wang and Dr Quanyi Wang (Department of Pathology, Affiliated Hospital of Jining Medical University), for their contributions to the scoring of immunohistochemistry.

\section{Funding}

The present study was supported by the Science and Technology Development Plan of Jining [grant no. (2016)56-086].

\section{Availability of data and materials}

The datasets used and/or analyzed during the present study are available from the corresponding author on reasonable request.

\section{Authors' contributions}

MG, XZ, and XH performed the experiments and wrote the manuscript. $\mathrm{XH}$ and LJ made substantial contributions to the conception and design of the manuscript. $\mathrm{XH}$ and $\mathrm{YZ}$ analyzed the experimental data. $\mathrm{MG}, \mathrm{XZ}, \mathrm{XH}$ and $\mathrm{YZ}$ assisted with the statistical analysis. MG and XH critically revised the manuscript and provided final approval of the version to be published. All authors read and approved the final version of the manuscript.

\section{Ethics approval and consent to participate}

Written informed consent was obtained from the patients and the study was approved by The Affiliated Hospital of Jining Medical University (Jining, China).

\section{Patient consent for publication}

Not applicable.

\section{Competing interests}

The authors declare that they have no competing interests.

\section{References}

1. Ferlay J, Soerjomataram I, Dikshit R, Eser S, Mathers C, Rebelo M, Parkin DM, Forman D and Bray F: Cancer incidence and mortality worldwide: Sources, methods and major patterns in GLOBOCAN 2012. Int J Cancer 136: E359-E386, 2015.

2. Siegel RL, Miller KD and Jemal A: Cancer statistics, 2018. CA Cancer J Clin 68: 7-30, 2018

3. Ciencewicki J, Trivedi S and Kleeberger SR: Oxidants and the pathogenesis of lung diseases. J Allergy Clin Immunol 122 456-468, 469-470, 2008

4. Ghio AJ, Carraway MS and Madden MC: Composition of air pollution particles and oxidative stress in cells, tissues, and living systems. J Toxicol Environ Health B Crit Rev 15: 1-21, 2012.

5. Höhn A, Jung T and Grune T: Pathophysiological importance of aggregated damaged proteins. Free Radic Biol Med 71: 70-89, 2014.

6. Barouki R: Ageing free radicals and cellular stress. Med Sci (Paris) 22: 266-272, 2006 (In French)
7. Ziech D, Franco R, Georgakilas AG, Georgakila S, Malamou-Mitsi V, Schoneveld O, Pappa A and Panayiotidis MI: The role of reactive oxygen species and oxidative stress in environmental carcinogenesis and biomarker development. Chem Biol Interact 188: 334-339, 2010.

8. Kryston TB, Georgiev AB, Pissis P and Georgakilas AG: Role of oxidative stress and DNA damage in human carcinogenesis. Mutat Res 711: 193-201, 2011.

9. Reuter S, Gupta SC, Chaturvedi MM and Aggarwal BB Oxidative stress, inflammation, and cancer: How are they linked? Free Radic Biol Med 49: 1603-1616, 2010.

10. Kardeh S, Ashkani-Esfahani S and Alizadeh AM: Paradoxical action of reactive oxygen species in creation and therapy of cancer. Eur J Pharmacol 735: 150-168, 2014.

11. Azimi I, Petersen RM, Thompson EW, Roberts-Thomson SJ and Monteith GR: Hypoxia-induced reactive oxygen species mediate $\mathrm{N}$-cadherin and SERPINE1 expression, EGFR signalling and motility in MDA-MB-468 breast cancer cells. Sci Rep 7: 15140, 2017.

12. Chiarugi P, Pani G, Giannoni E, Taddei L, Colavitti R, Raugei G, Symons M, Borrello S, Galeotti T and Ramponi G: Reactive oxygen species as essential mediators of cell adhesion: The oxidative inhibition of a FAK tyrosine phosphatase is required for cell adhesion. J Cell Biol 161: 933-944, 2003.

13. Poillet-Perez L, Despouy G, Delage-Mourroux R and Boyer-Guittaut M: Interplay between ROS and autophagy in cancer cells, from tumor initiation to cancer therapy. Redox Biol 4: 184-192, 2015.

14. Itkonen $\mathrm{O}$ and Stenman UH: TATI as a biomarker. Clin Chim Acta 431: 260-269, 2014.

15. Stenman UH: Role of the tumor-associated trypsin inhibitor SPINK1 in cancer development. Asian J Androl 13: 628-629, 2011.

16. Räsänen K, Itkonen O, Koistinen $\mathrm{H}$ and Stenman UH: Emerging Roles of SPINK1 in Cancer. Clin Chem 62: 449-457, 2016.

17. Stenman UH: SPINK1: A new therapeutic target in cancer? Clin Chem 57: 1474-1475, 2011.

18. Edge SB and Compton CC: The American Joint Committee on Cancer: The 7th edition of the AJCC cancer staging manual and the future of TNM. Ann Surg Oncol 17: 1471-1474, 2010.

19. Jiao F, Hu H, Han T, Zhuo M, Yuan C, Yang H, Wang L and Wang L: Aberrant expression of nuclear HDAC 3 and cytoplasmic $\mathrm{CDH} 1$ predict a poor prognosis for patients with pancreatic cancer. Oncotarget 7: 16505-16516, 2016.

20. Peirson SN, Butler JN and Foster RG: Experimental validation of novel and conventional approaches to quantitative real-time PCR data analysis. Nucleic Acids Res 31: e73, 2003.

21. Livak KJ and Schmittgen TD: Analysis of relative gene expression data using real-time quantitative PCR and the 2(-Delta Delta C(T)) method. Methods 25: 402-408, 2001.

22. Kwak MK, Kensler TW and Casero RJ Jr: Induction of phase 2 enzymes by serum oxidized polyamines through activation of Nrf2: Effect of the polyamine metabolite acrolein. Biochem Biophys Res Commun 305: 662-670, 2003.

23. Kong $\mathrm{H}$ and Chandel NS: Regulation of redox balance in cancer and T cells. J Biol Chem 293: 7499-7507, 2018.

24. Liang C, Shi S, Liu M, Qin Y, Meng Q, Hua J, Ji S, Zhang Y, Yang $\mathrm{J}, \mathrm{Xu} \mathrm{J}$, et al: PIN1 maintains redox balance via the c-Myc/NRF2 axis to counteract Kras-induced mitochondrial respiratory injury in pancreatic cancer cells. Cancer Res 79: 133-145, 2019.

25. Masamune A, Mizutamari H, Kume K, Asakura T, Satoh K and Shimosegawa T: Hereditary pancreatitis as the premalignant disease: A Japanese case of pancreatic cancer involving the SPINK1 gene mutation N34S. Pancreas 28: 305-310, 2004.

26. Ohmuraya M, Hirota M, Araki M, Mizushima N, Matsui M, Mizumoto T, Haruna K, Kume S, Takeya M, Ogawa M, et al: Autophagic cell death of pancreatic acinar cells in serine protease inhibitor Kazal type 3-deficient mice. Gastroenterology 129: 696-705, 2005

27. Ying HY, Gong CJ, Feng Y, Jing DD and Lu LG: Serine protease inhibitor Kazal type 1 (SPINK1) downregulates E-cadherin and induces EMT of hepatoma cells to promote hepatocellular carcinoma metastasis via the MEK/ERK signaling pathway. J Dig Dis 18: 349-358, 2017.

28. Gaber A, Stene C, Hotakainen K, Nodin B, Palmquist I, Bjartell A, Stenman UH, Jeppsson B, Johnson LB and Jirström K: Effects of radiation therapy on tissue and serum concentrations of tumour associated trypsin inhibitor and their prognostic significance in rectal cancer patients. Radiat Oncol 6: 100, 2011. 
29. Moloney JN and Cotter TG: ROS signalling in the biology of cancer. Semin Cell Dev Biol 80: 50-64, 2018.

30. Prasad S, Gupta SC and Tyagi AK: Reactive oxygen species (ROS) and cancer: Role of antioxidative nutraceuticals. Cancer Lett 387: 95-105, 2017.

31. Bapat S, Verkleij A and Post JA: Peroxynitrite activates mitogen-activated protein kinase (MAPK) via a MEK-independent pathway: A role for protein kinase C. Febs Lett 499: 21-26, 2001.

32. Farrukh MR, Nissar UA, Afnan Q, Rafiq RA, Sharma L, Amin S, Kaiser P, Sharma PR and Tasduq SA: Oxidative stress mediated $\mathrm{Ca}(2+)$ release manifests endoplasmic reticulum stress leading to unfolded protein response in UV-B irradiated human skin cells. J Dermatol Sci 75: 24-35, 2014.

33. Panayiotidis M: Reactive oxygen species (ROS) in multistage carcinogenesis. Cancer Lett 266: 3-5, 2008

34. Miller JH, Jin S, Morgan WF, Yang A, Wan Y, Aypar U, Peters JS and Springer DL: Profiling mitochondrial proteins in radiation-induced genome-unstable cell lines with persistent oxidative stress by mass spectrometry. Radiat Res 169: 700-706, 2008.

35. Zong WX, Rabinowitz JD and White E: Mitochondria and cancer. Mol Cell 61: 667-676, 2016.

36. Chen J, Zhang L, Yu X, Zhou H, Luo Y, Wang W and Wang L: Clinical application of plasma mitochondrial DNA content in patients with lung cancer. Oncol Lett 16: 7074-7081, 2018.

37. Sansone P, Savini C, Kurelac I, Chang Q, Amato LB, Strillacci A Stepanova A, Iommarini L, Mastroleo C, Daly L, et al: Packaging and transfer of mitochondrial DNA via exosomes regulate escape from dormancy in hormonal therapy-resistant breast cancer. Proc Natl Acad Sci USA 114: E9066-E9075, 2017.

38. Lin CS, Liu LT, Ou LH, Pan SC, Lin CI and Wei YH: Role of mitochondrial function in the invasiveness of human colon cancer cells. Oncol Rep 39: 316-330, 2018.

39. DeGraff WG, Krishna MC, Kaufman D and Mitchell JB Nitroxide-mediated protection against X-ray- and neocarzinostatin-induced DNA damage. Free Radic Biol Med 13: 479-487, 1992

40. Zhou D, Shao L and Spitz DR: Reactive oxygen species in normal and tumor stem cells. Adv Cancer Res 122: 1-67, 2014.
41. Yu J, Sun W, Song Y, Liu J, Xue F, Gong K, Yang X and Kang Q: SIRT6 protects retinal ganglion cells against hydrogen peroxide-induced apoptosis and oxidative stress by promoting Nrf2/ARE signaling via inhibition of Bach1. Chem Biol Interact 300: 151-158, 2019.

42. Sajadimajd S and Khazaei M: Oxidative stress and cancer: The role of Nrf2. Curr Cancer Drug Targets 18: 538-557, 2018.

43. Deshmukh P, Unni S, Krishnappa G and Padmanabhan B: The Keap1-Nrf2 pathway: Promising therapeutic target to counteract ROS-mediated damage in cancers and neurodegenerative diseases. Biophys Rev 9: 41-56, 2017.

44. Taguchi K and Yamamoto M: The KEAP1-NRF2 system in cancer. Front Oncol 7: 85, 2017.

45. Hayes AJ, Skouras C, Haugk B and Charnley RM: Keap1-Nrf2 signalling in pancreatic cancer. Int J Biochem Cell Biol 65: 288-299, 2015.

46. Ozaki N, Ohmuraya M, Hirota M, Ida S, Wang J, Takamori H, Higashiyama S, Baba H and Yamamura K: Serine protease inhibitor Kazal type 1 promotes proliferation of pancreatic cancer cells through the epidermal growth factor receptor. Mol Cancer Res 7: 1572-1581, 2009.

47. Wang C, Wang L, Su B, Lu N, Song J, Yang X, Fu W, Tan W and Han B: Serine protease inhibitor Kazal type 1 promotes epithelial-mesenchymal transition through EGFR signaling pathway in prostate cancer. Prostate 74: 689-701, 2014

48. Chen YT, Tsao SC, Yuan SS, Tsai HP and Chai CY: Serine protease inhibitor Kazal type 1 (SPINK1) promotes proliferation of colorectal cancer through the epidermal growth factor as a prognostic marker. Pathol Oncol Res 21: 1201-1208, 2015.

49. Ateeq B, Tomlins SA, Laxman B, Asangani IA, Cao Q, Cao X, Li Y, Wang X, Feng FY, Pienta KJ, et al: Therapeutic targeting of SPINK1-positive prostate cancer. Sci Transl Med 3: 72ra17, 2011.

This work is licensed under a Creative Commons Attribution-NonCommercial-NoDerivatives 4.0 International (CC BY-NC-ND 4.0) License. 